\title{
Mapping Green Spaces in Bishkek-How Reliable can Spatial Analysis Be?
}

\section{Peter Hofmann $^{1,2, *}$, Josef Strobl ${ }^{1,2,3}$ and Ainura Nazarkulova ${ }^{3}$}

1 Department of GIScience, Austrian Academy of Sciences, Schillerstr. 30, A-5020 Salzburg, Austria

2 Centre for Geoinformatics, University of Salzburg, Hellbrunnerstr. 34, A-5020 Salzburg, Austria;

E-Mail: josef.strobl@sbg.ac.at

3 Austria-Central Asia Centre for GIScience, Maldybaeva Street 34 “B”, Bischkek 720020,

Kyrgyzstan; E-Mail: nazarkulova@aca-giscience.org

* Author to whom correspondence should be addressed; E-Mail: peter.hofmann@oeaw.ac.at;

Tel.: +43-662-8044-7514; Fax: +43-662-8044-5260.

Received: 19 April 2011; in revised form: 16 May 2011 / Accepted: 17 May 2011 /

Published: 30 May 2011

\begin{abstract}
Within urban areas, green spaces play a critically important role in the quality of life. They have remarkable impact on the local microclimate and the regional climate of the city. Quantifying the 'greenness' of urban areas allows comparing urban areas at several levels, as well as monitoring the evolution of green spaces in urban areas, thus serving as a tool for urban and developmental planning. Different categories of vegetation have different impacts on recreation potential and microclimate, as well as on the individual perception of green spaces. However, when quantifying the 'greenness' of urban areas the reliability of the underlying information is important in order to qualify analysis results. The reliability of geo-information derived from remote sensing data is usually assessed by ground truth validation or by comparison with other reference data. When applying methods of object based image analysis (OBIA) and fuzzy classification, the degrees of fuzzy membership per object in general describe to what degree an object fits (prototypical) class descriptions. Thus, analyzing the fuzzy membership degrees can contribute to the estimation of reliability and stability of classification results, even when no reference data are available. This paper presents an object based method using fuzzy class assignments to outline and classify three different classes of vegetation from GeoEye imagery. The classification result, its reliability and stability are evaluated using the reference-free parameters Best Classification Result and Classification Stability as introduced by Benz et al. in 2004 and implemented in the software package eCognition
\end{abstract}


(www.ecognition.com). To demonstrate the application potentials of results a scenario for quantifying urban 'greenness' is presented.

Keywords: Object Based Image Analysis; GeoEye; urban green; fuzzy classification; classification reliability

\section{The Role of Green Spaces in Bishkek}

Although embedded in an area with semi-arid climate, the capital of Kyrgyzstan is widely recognized and labeled as a 'green city'. Bishkek's mostly tree-lined streets, parks and other urban green areas are maintained through hot summers by a network of open irrigation channels. This lush vegetation essentially is the only 'green' factor of the city and contributes substantially to the quality of life of Bishkek's residents. As ascertained by [1] and [2], vegetation affects urban climate by moderating temperature, increasing humidity, influencing wind speed and reducing noise. Further desirables are reduction of solar radiation, view screening and visual amenity. Since green spaces are not distributed evenly throughout the city, the spatial distribution and density of urban green spaces is of interest for city planners as well as for real estate developers and of course for individuals looking for attractive residential and business locations. The methodology outlined in this paper therefore can provide decision support and planning assistance for these target groups, as well as create input data for urban climate modeling as outlined in [3].

\section{Methods and Objectives}

In general, GIS acts as a key tool for the integration and leverage of geo-referenced information for planning, decision making and assessment. In this context the objectives of this study are: (a) to generate a transferable and flexibly applicable methodology for mapping urban green spaces based on remote sensing data; (b) to define indices for rating recreational potential and other factors on a regionalized basis; (c) to develop a framework for enabling the monitoring of green spaces quantitatively and qualitatively on the basis of the Green Index as outlined in [4]; and (d) to offer methods to assess the reliability of spatial analysis results based upon the underlying image analysis results. Since vegetation is a relatively dynamic land cover class, methods of detecting its physical and spatial conditions over a larger (urban) area and over longer periods (synoptically) are proposed through the analysis of remote sensing data. With respect to the complex and fine-grained structures of urban areas, remote sensing data with appropriate spatial and radiometric capabilities have to be used. For a more differentiated determination of the Green Index, a rough categorization of vegetation (e.g., grassy vs. wooded) is an asset. In the example presented here, different vegetation types detected from remote sensing data act as weighted input for determining the 'greenness' of a region. Since the reliability and stability of the image classification directly affects the reliability of the calculated Green Index, this is calculated and visualized respectively. 


\section{Detecting Urban Green Spaces from GeoEye-1 Data}

Throughout this investigation, we have used a subset of a GeoEye-1 image fulfilling the Geo ${ }^{\mathrm{TM}}$ product standards of GeoEye (http://www.geoeye.com/CorpSite/products/), covering the southern part of Bishkek. The image was acquired on 16 August 2009 with zero percent cloud coverage. During this capture time in the region grassy vegetation is usually completely dry, while trees, bushes and areas under irrigation can be observed as green. Consequently, the near infrared (NIR) signal of dry grassy vegetation is reduced and similar to that of non-vegetation land cover classes. In addition, a quick inspection shows several locations with extreme blooming effects resulting from intense reflections at plane (roof) surfaces.

\subsection{Pre-Processing}

In order to fully benefit from the data's spatial and spectral capabilities we were pan-sharpening the subset by applying the principal components method as suggested in [5] (Figure 1). Additionally, for further analysis the NDVI (Normalized Difference Vegetation Index, [6]) has been calculated on the pan-sharpened subset per pixel and used as an additional channel (Figure 2).

Figure 1. Subset of area under investigation from GeoEye-1 data. Original data pan-sharpened (see text for details) with a vegetation-denoting color visualization $($ red $=$ red, green $=($ green $+\mathrm{NIR}) / 2$ and blue $=$ blue $)$.

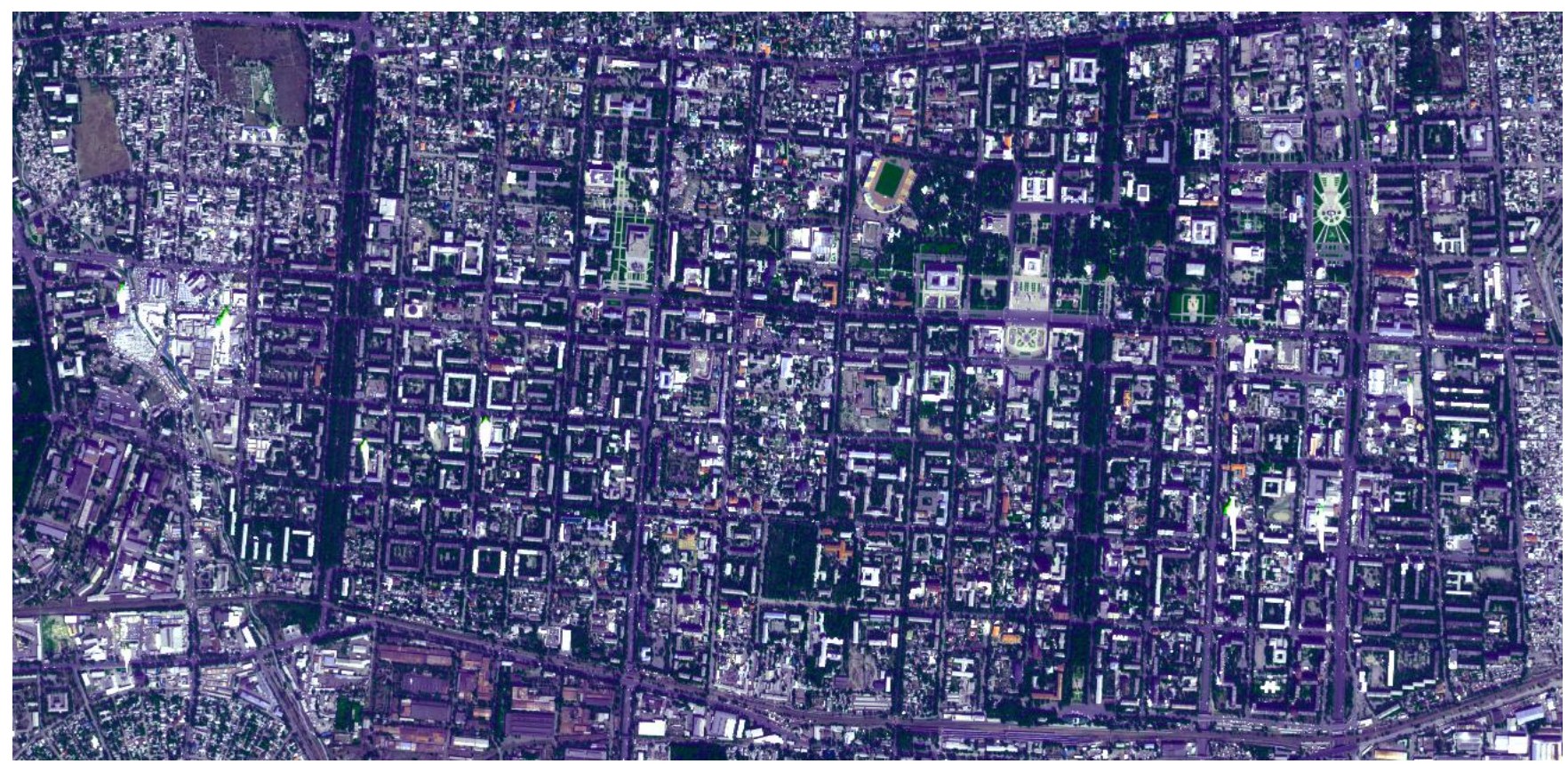


Figure 2. Calculated NDVI for subset area based on GeoEye-1 data.

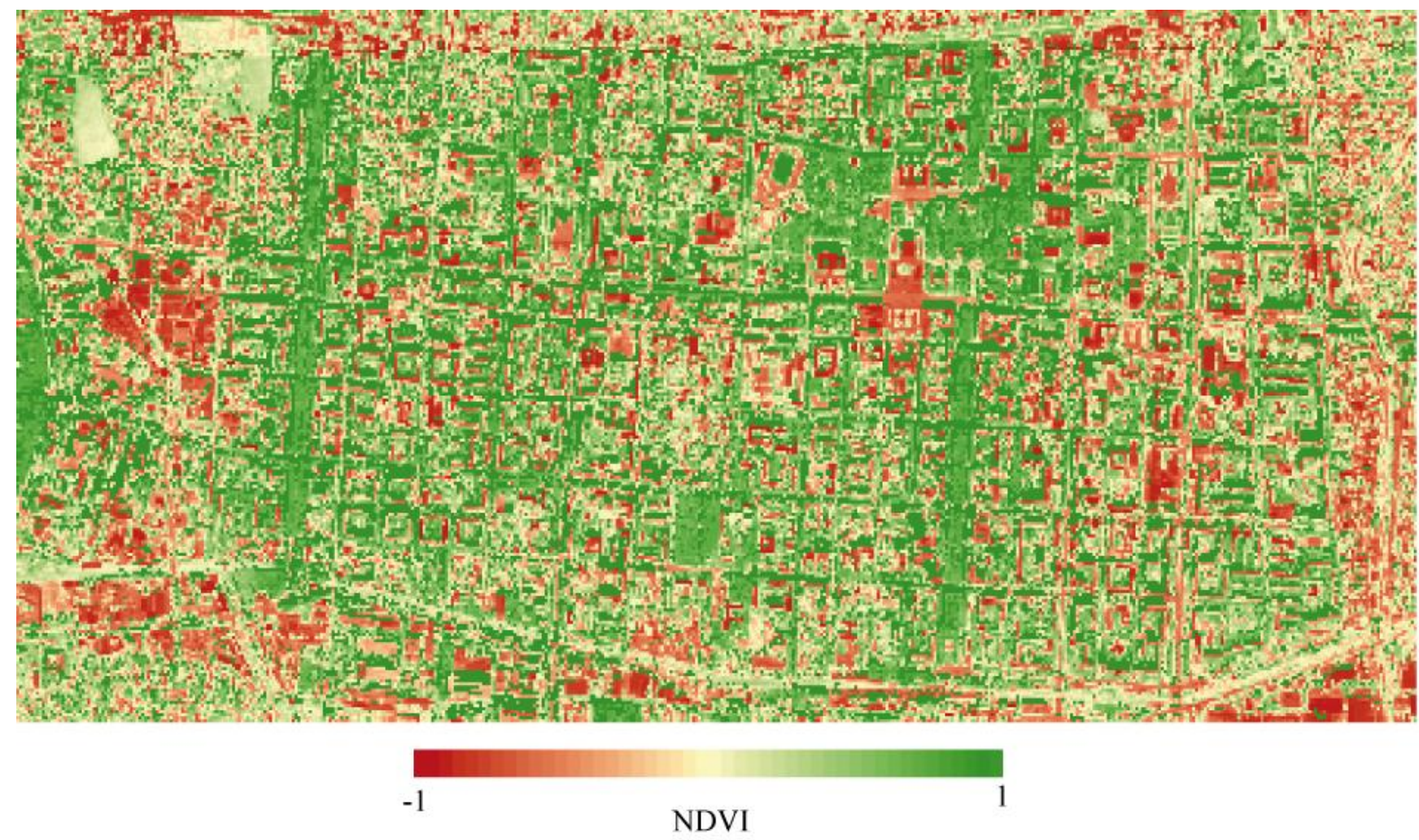

\subsection{Object Based Image Analysis}

For detecting and further differentiating vegetation we followed the approach of object based image analysis (OBIA) [7]. OBIA as a method for image analysis has evolved in the last decade, especially for analyzing remote sensing data with high spatial resolution. In comparison to per-pixel-based methods of image analysis, OBIA uses image objects instead of pixels as the building blocks for image classification. These image objects are generated by arbitrary, knowledge-free image segmentation, whereas the segmentation process is usually steered by one or more homogeneity criteria concerning color and shape which have to be parameterized [8-10]. Recognized major advantages of OBIA are the reduction of noise and the extension of the potential feature space [11-14]. That is, instead of per-pixel-feature-values aligned in a layer-stack-like manner, objects can be analyzed and classified based upon their statistical spectral features, their texture and their shape. Linking the generated objects, topological relations between objects can be used for image analysis in a manner typical for GIS. This way it is even possible to describe and use spatial context information, such as neighborhood relations and distances. Some researchers $[15,16]$ name the potential to use concepts of scale [17] and mereology through a hierarchical network of image objects as a further advantage of OBIA. Because of these GIS-like characteristics used in image analysis, OBIA is often considered as the bridging element between remote sensing and GIS $[18,19]$. In order to assign the generated image objects to classes of their corresponding real-world objects, in principle any sensible classification method can be used. Without going into details about classification methods, widely used classification methods in OBIA are: (a) rule-based methods which classify objects according to expert knowledge formulated in rules [20-22]; and (b) sample-based methods which assign objects to classes according to their similarity to samples, that is, their distance from samples in feature space [23,24]. Both principles can 
be applied using so-called hard or soft classifiers, that is, assigning objects to distinct classes (hard classifiers, such as threshold-based assignment) or allow objects to be a gradual member of more than one class (soft classifiers, such as fuzzy classifiers or neural networks [22,25]). The last case only makes sense in conjunction with respective expressions for the gradual class assignment per object. In the present case, we were using the software package eCognition 8 Developer (http://www.ecognition.com) for OBIA. We first applied a multi resolution segmentation [10] which is a global region growing method mainly controlled by the so-called 'scale parameter' determining the maximum allowed heterogeneity of the segments to be created. The scale parameter is constituted by the weighted heterogeneity of color and shape, whereas the heterogeneity of shape is constituted by weighting compactness $v s$. smoothness. Compactness is defined by the ratio of a segment's perimeter $P_{O b j}$ to its area $A_{O b j}$; smoothness is defined by the ratio of the object's perimeter to the perimeter of its minimum bounding box parallel to the image grid $P_{M B B}$. Both together form the shape homogeneity $h_{\text {form }}$ by weighting them to the sum of 1 :

$$
h_{f o r m}=w \cdot \frac{P_{O b j}}{P_{M B B}}+(1-w) \cdot \frac{P_{O b j}}{A_{O b j}}
$$

with $w \in R^{+}$and $0 \leq w \leq 1$. The heterogeneity of color $h_{\text {color }}$ is defined by the weighted sum of the segment's standard deviations per channel:

$$
h_{\text {color }}=\sum_{c=1}^{n} w_{c} \sigma_{c}
$$

with $w_{c} \in R^{+}$and $0 \leq w \leq 1$ the weight of channel number $c$ and $\sigma_{c}$ the standard deviation of the segment's pixels in channel $c$. Neighboring segments or pixels are merged if their weighted combined color and shape heterogeneity $h$ :

$$
h=w \cdot h_{\text {color }}+(1-w) \cdot h_{\text {form }}
$$

with $w \in R^{+}$and $0 \leq w \leq 1$ is a minimum and below the scale parameter (see [10] and [20] for details). We applied the multi resolution segmentation on the four pan-sharpened channels with a scale parameter of 100 and a weighting of 0.9 for color and 0.1 for shape. Compactness and smoothness were weighted by 0.5 each and each channel was weighted equally (Figure 3 ).

In order to mask blooming effects, we classified all segments with an average brightness of more than 1,500 respectively. For the next classification steps, we applied a fuzzy hierarchical classification scheme [26]. Hierarchical means: classes are sorted into sub- and super-classes by their common (super-class) and individual (sub-class) properties. This way, sub-classes inherit the properties of their super-classes. That is, all sub-classes share the class-description of their super-class (Figure 4.). Simultaneously, classes can also be sorted following a semantic hierarchy scheme. That is, classes with similar meaning can be pooled and labeled by a common semantic super-class, although their physical properties might be very different. These common semantic labels can be used for the description and analysis of topological relationships. 
Figure 3. Segmentation result from multi resolution segmentation (see text for details) zoomed into the red marked zone in the north-east.

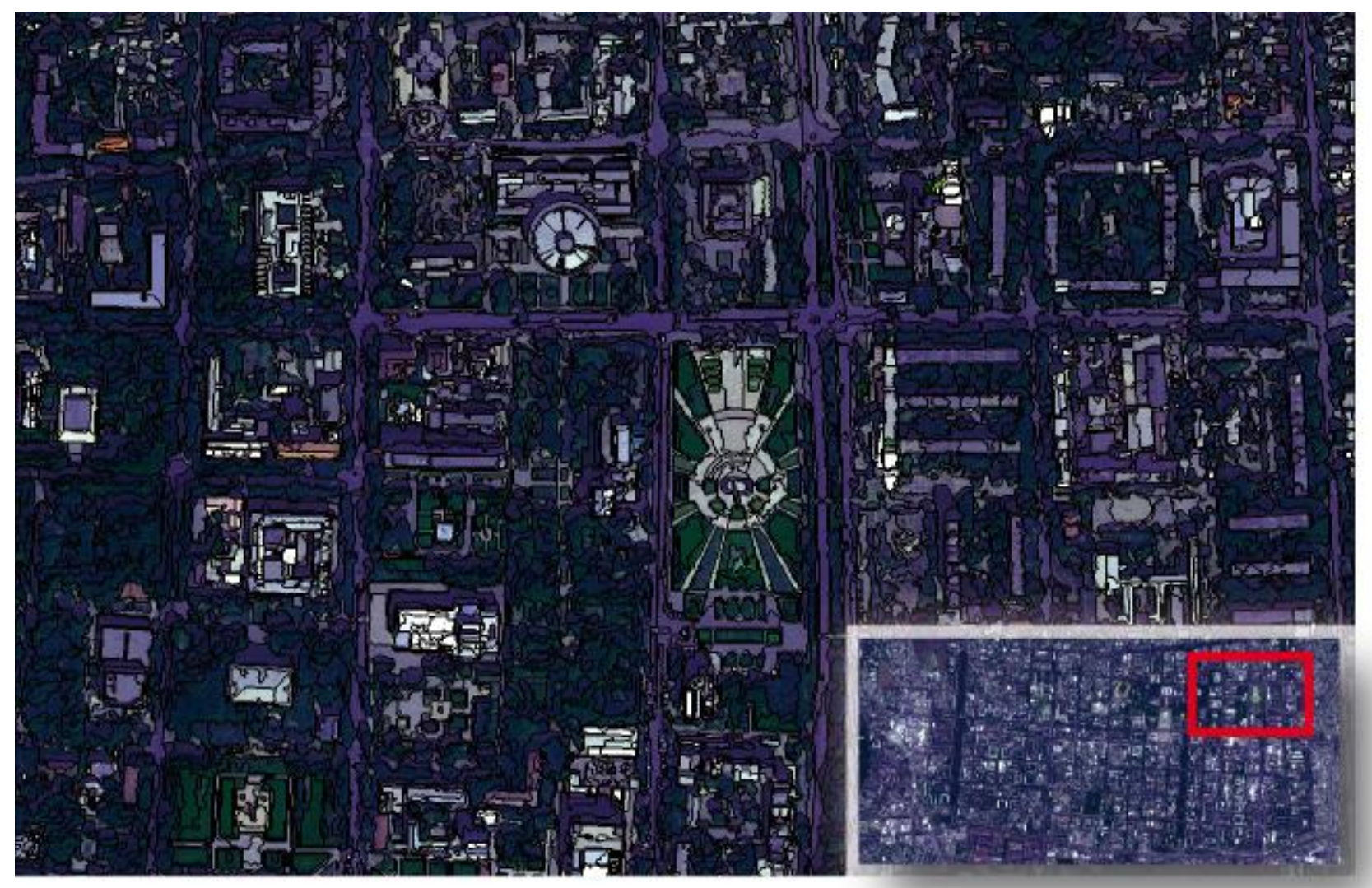

Figure 4. Inheritance hierarchy of vegetation classes (left) and exemplary ('meadow-like vegetation') class description by fuzzy-membership functions and respective fuzzy operators (right). The semantic hierarchy looks similar to the inheritance hierarchy.

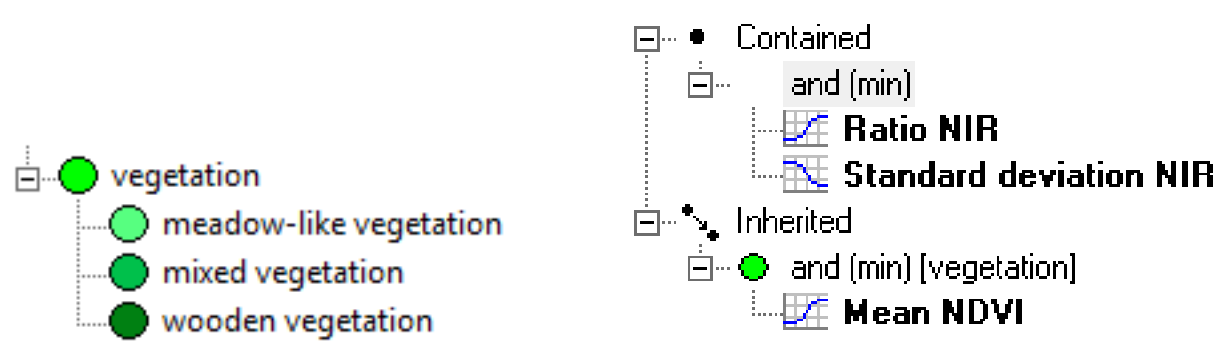

Each class of this scheme can be described as a fuzzy set within feature space (see [20] and [27]). That is, instead of crisp class assignment, each object obtains a degree of membership $\mu$ with $\mu \in R^{+}$ and $0 \leq \mu \leq 1$ to one or more classes. This way, $\mu$ expresses for each object its degree of fulfilling the classification conditions for each individual class in a range between 0 and 1 . When using more than one feature to describe the class membership of an object, $\mu$ is the result of the fuzzy combinations of the membership degrees concerning these features. That is, the object's individual degree of membership is the result of a fuzzy combination of membership functions connected via the operators fuzzy-AND (returning the minimum $\mu$ for all properties) and fuzzy-OR (returning the maximum $\mu$ for all properties). Fuzzy membership functions can be of different shape depending on how to express $\mu$ concerning the property used (see Figure 5). 
Figure 5. Rule set consisting of classes $A$ and $B$ described by fuzzy membership functions concerning features $a, b, c, d, e, f$ which are connected via fuzzy-and and fuzzy-or operators.
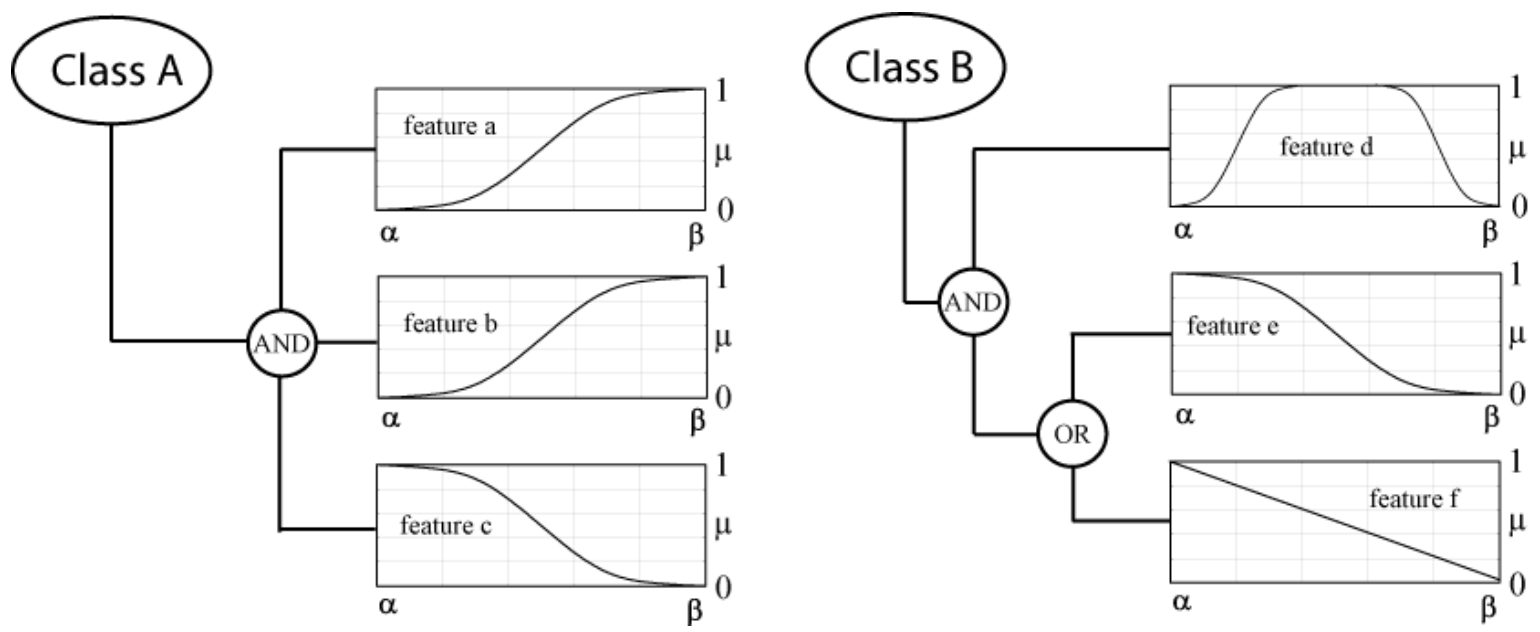

The upper border of a membership function along the feature value axis is usually named $\beta$ and the lower border is usually named $\alpha$. That is, for a fuzzy-greater-than function-as like the membership functions concerning feature $a$ and $b$ in Figure 5- $\mu=1.0$ at $a=\beta$ and $b=\beta$ and $\mu=0.0$ at $a=\alpha$ and $b=\alpha$. Vice versa for a fuzzy-lower-than function (e.g., feature $c, e$ and $f$ in Figure 5). A fuzzy-range function combines a fuzzy-lower-than and fuzzy-greater-than function in a single membership function (feature $d$ in Figure 5). Hence, $\mu$ is at maximum in the range of the upper bound of the greater-than part and the lower bound of the lower-than part of the range function. Combinations with a single maximum at $\alpha+((\beta-\alpha) / 2)$ are possible, too. Although individual shapes of membership functions are possible in principle, the shapes outlined here are most common, since they are easy to understand and therefore make the interpretation of fuzzy classification results more comprehensive. For example, the class descriptions depicted in Figure 5 can be interpreted as follows:

- object $i$ is the more a member of class $A$, the closer its value of feature $a$ and $b$ is to $\beta$ and the closer its value for feature $c$ is to $\alpha$.

- the final degree of membership to class $A$ is the minimum membership value of the membership functions for feature $a, b$ and $c$ :

$$
\mu_{i}^{A}=\min \left(\mu_{i}^{a}, \mu_{i}^{b}, \mu_{i}^{c}\right)
$$

- the lower the value of feature $f$ or $e$ for object $i$ is and the closer its value of feature $d$ lies in the range between $\alpha$ and $\beta$, the more object $i$ belongs to class $B$ :

$$
\mu_{i}^{B}=\min \left(\mu_{i}^{d}, \max \left(\mu_{i}^{e}, \mu_{i}^{f}\right)\right)
$$

Note: an individual object $i$ can be a member of more than one class but with different degrees of membership, describing the ambiguity of a fuzzy classification result. In practice, when de-fuzzyfying the fuzzy classification result, object $i$ is crisply assigned to the class with the maximum degree of membership above a to-be-defined threshold. For $n \in N$ classes, the membership degree in the 'best' class is defined as Best Classification Result $\mu_{i}^{b}$ for object $i$ (see [20]): 


$$
\mu_{i}^{b}=\max \left(\mu_{i}^{1}, \ldots, \mu_{i}^{n}\right)
$$

Within the class hierarchy, in the case presented, the class 'vegetation' acts as the super-class for 'wooded vegetation', 'meadow-like vegetation' and 'mixed vegetation' (Figure 4). Consequently, these sub-classes inherit the NDVI-description of 'vegetation'. For each of the sub-classes the fuzzy description concerning the mean NDVI is connected with its individual descriptions by a fuzzy-AND operator (Figure 4). In our particular case, the classes were described as depicted in Table 1 producing the classification result as displayed in (Figure 6).

Table 1. Fuzzy class descriptions of vegetation classes.

\begin{tabular}{|c|c|c|c|c|}
\hline \multirow{2}{*}{ Class } & \multirow{2}{*}{ Property } & \multirow{2}{*}{ Membership Function } & \multicolumn{2}{|c|}{ Parameters of Membership Function } \\
\hline & & & $\alpha$ & $\beta$ \\
\hline \multirow[t]{2}{*}{ vegetation } & Mean NDVI & & 0.45 & 0.60 \\
\hline & Ratio NIR & 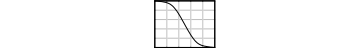 & 0.40 & 0.50 \\
\hline \multirow[t]{2}{*}{ wooded vegetation } & $\begin{array}{c}\text { Standard Dev. } \\
\text { NIR }\end{array}$ & $E$ & 35.00 & 50.00 \\
\hline & Ratio NIR & E & 0.40 & 0.70 \\
\hline \multirow[t]{2}{*}{ meadow-like vegetation } & $\begin{array}{c}\text { Standard Dev. } \\
\text { NIR }\end{array}$ & $\otimes$ & 45.00 & 65.00 \\
\hline & Ratio NIR & $\theta$ & 0.45 & 0.75 \\
\hline mixed vegetation & $\begin{array}{c}\text { Standard Dev. } \\
\text { NIR }\end{array}$ & E & 30.00 & 50.00 \\
\hline
\end{tabular}

Figure 6. Classification results superimposed on pan-sharpened image data, differentiating three vegetation classes.
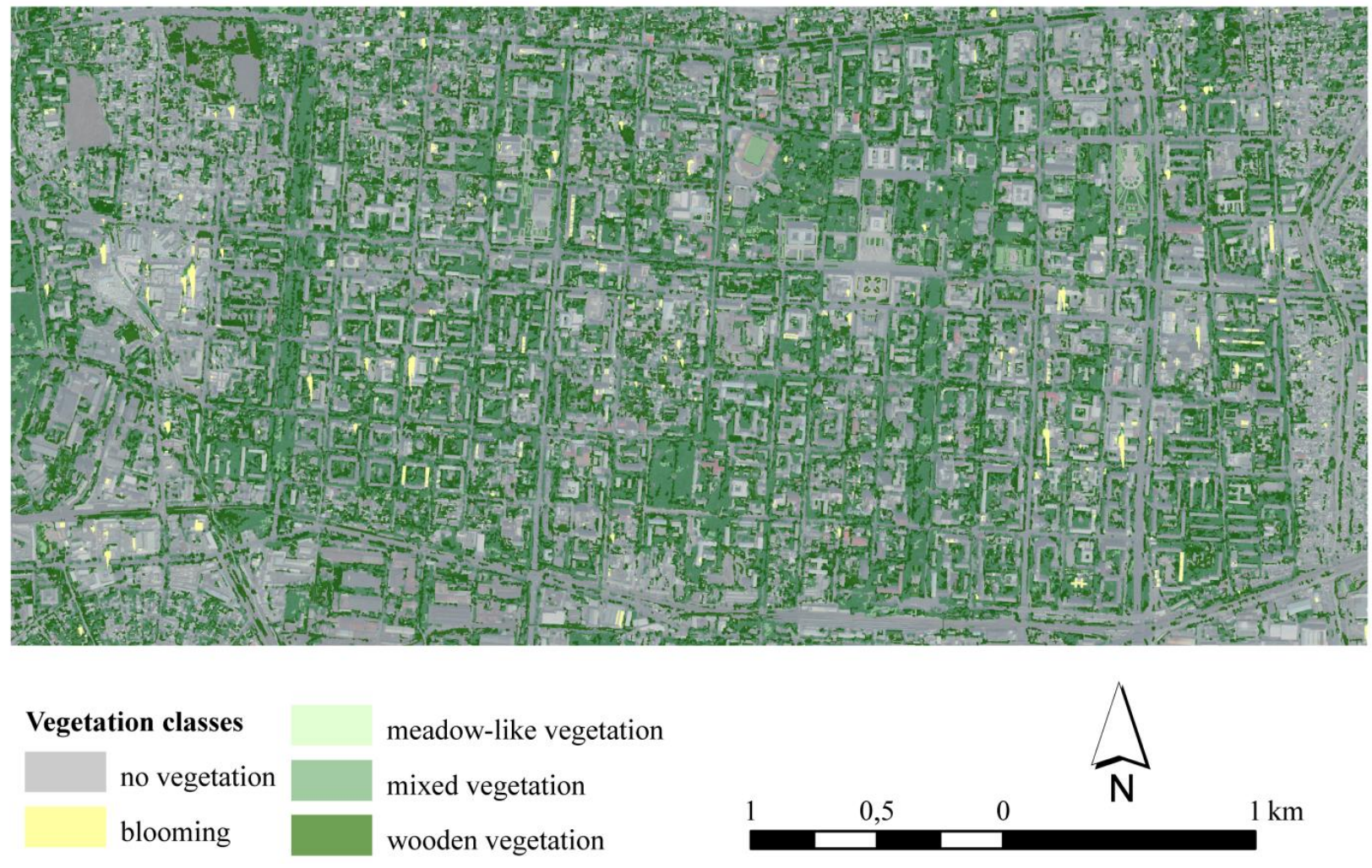
Their spectral properties were described by the color fraction (ratio) of the NIR channel only. According to [27] the ratio of a channel within an object is defined as follows: Let $b_{i}^{c}$ be the mean value $(D N)$ of an object with $p$ pixels in channel $c$ :

$$
b_{i}^{c}=\frac{1}{p} \cdot \sum_{j=1}^{p} D N_{j}^{c}
$$

The overall brightness $b_{i}$ of an object is defined as the weighted mean over all channels of an object:

$$
b_{i}=\frac{1}{n} \cdot \sum_{j=1}^{n} w_{j} b_{i}^{j}
$$

with $w_{j} \in R^{+}$and $0 \leq w_{j} \leq 1$ the weight of channel $j$. The ratio $r_{i}^{c}$ of channel $c$ in object $i$ is defined as:

$$
r_{i}^{c}=\frac{b_{i}^{c}}{b_{i}}
$$

whereas $r_{i}^{c}=0$ if $b_{i}=0$ or $w_{c}=0$ respectively. The standard deviation per object in the NIR channel describes the spectral homogeneity of an object concerning this particular feature. The lower the standard deviation, the more spectrally homogeneous an object is considered and vice versa. Thus, the standard deviation is rather a texture describing feature than a spectral characteristic.

A side effect of using a hierarchical classification approach is the handling of objects fulfilling the criteria of super-classes but none of the respective sub-classes. If there is no explicit alternative sub-class defined (which is expressed as the inverse of all other sub-classes), objects fulfilling the criteria of a super-class but none of a sub-class remain unclassified. However, such an alternative sub-class has the disadvantage of semantically being a rather diffuse class (usually named as "others" or "rest"). Hence, we did not create such an alternative vegetation sub-class, which led to some unclassified vegetation objects (Tables 2 and 3).

Table 2. Global Statistics for Best Classification Result $\left(\mu_{i}^{b}\right)$.

\begin{tabular}{lccccc}
\hline Class & No. of Objects & Mean & Standard Deviation & Min. & Max. \\
\hline vegetation & 18,748 & 0.87 & 0.26 & 0.10 & 1.00 \\
After classifying vegetation child classes & & & & \\
\hline Class & No. of Objects & Mean & Standard Deviation & Min. & Max. \\
\hline wooded vegetation & 9,232 & 0.65 & 0.30 & 0.10 & 1.00 \\
meadow-like vegetation & 644 & 0.84 & 0.22 & 0.10 & 1.00 \\
mixed vegetation & 8,003 & 0.86 & 0.21 & 0.11 & 0.99 \\
\hline
\end{tabular}

Table 3. Global Statistics for Classification Stability $\left(C S_{i}\right)$.

\begin{tabular}{lccccc}
\hline Class & No. of Objects & Mean & Standard Deviation & Min. & Max. \\
\hline vegetation & 18,748 & 0.87 & 0.26 & 0.10 & 1.00 \\
After classifying vegetation child classes & & & & \\
\hline Class & No. of Objects & Mean & Standard Deviation & Min. & Max. \\
\hline wooded vegetation & 9,232 & 0.64 & 0.32 & 0.00 & 1.00 \\
meadow-like vegetation & 644 & 0.47 & 0.35 & 0.00 & 1.00 \\
mixed vegetation & 8,003 & 0.72 & 0.30 & 0.00 & 1.00 \\
\hline
\end{tabular}


As the class descriptions show, the sub-classes are hard to separate, due to some degree of overlap in feature space. Thus, a clear and distinct assignment of vegetation objects to one of the three child classes for some objects is hardly feasible. These objects then are member of more than one class, but to different degrees of membership. This ambiguity is expressed by the Classification Stability (see [20] and [26]) per object $\left(C S_{i}\right)$, taking into account the fuzzy membership of an object to multiple classes:

$$
C S_{i}=\mu_{i}^{b}-\mu_{i}^{s}
$$

with $\mu_{i}^{b}$ as the Best Classification Result for object $i$ to the class it was assigned and $\mu_{i}^{s}$ the degree of fuzzy membership in the class object $i$ fulfills the classification criteria at second-best level, with $\mu_{i}^{b} \geq \mu_{i}^{s}$ and $\mu_{i}^{b}, \mu_{i}^{s} \in[0,1]$. That means, object $i$ is a member of the second-best class, too, but to the lower membership degree of $\mu_{i}^{s}$. The higher $\mu_{i}^{b}$, the better object $i$ satisfies the classification criteria of the class it was assigned to. The higher $C S_{i}$, the less ambiguous an object $i$ is classified and the less it belongs to the second-best class respectively. Since $\mu_{i}^{b}$ and $C S_{i}$ express how distinctly an object belongs to the class it was assigned to, both values express the reliability of the crisp class assignment after de-fuzzyfication (Figure 7).

Figure 7. Interrelationship between $C S_{i}$ (red indicates low, green indicates high value for $\left.C S_{i}\right), \mu_{i}^{b}$ and $\mu_{i}^{s}$.

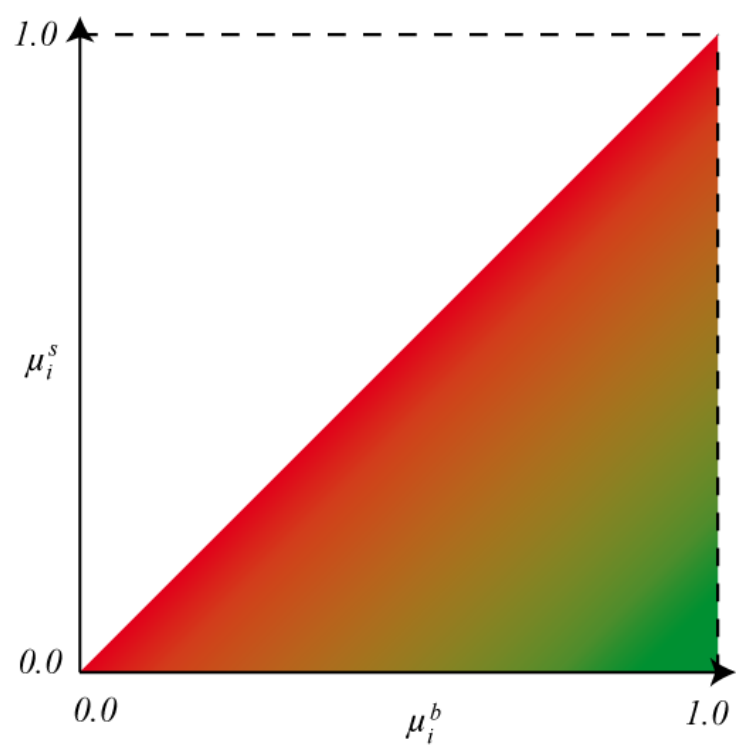

Analyzing statistical moments, such as mean and standard deviation of $C S_{i}$ and $\mu_{i}^{b}$ of the whole scene can be helpful in terms of assessing global reliability and adequacy of class descriptions (Tables 2 and 3 ; see $[26,27])$.

Table 2 indicates that objects of the super-class 'vegetation' fulfill the classification criteria on average by 0.87 .869 'vegetation' objects $(18748-(9232+644+8003)=869)$ could not further be assigned to any of its sub-classes since they do not fulfill any of the respective classification conditions. Objects of the class 'mixed vegetation' were classified most distinctly, but there is no object of this class being a full member of it (maximum $\mu_{i}^{b}=0.99$ ). 'Meadow-like vegetation' obviously is least separable from other classes (mean $C S_{i}=0.47$ ). A map-like display of $C S_{i}$ and $\mu_{i}^{b}$ per object shows the spatial distribution of the values and can reveal spatial concentrations of (un)ambiguity (Figure 8). 
Figure 8. Reliability of classification results per object expressed by Best Classification Result $\left(\mu_{i}^{b}\right)$ per object (top) and Classification Stability $\left(C S_{i}\right)$ per object bottom. Both superimposed to GeoEye-1 pan-sharpened image.

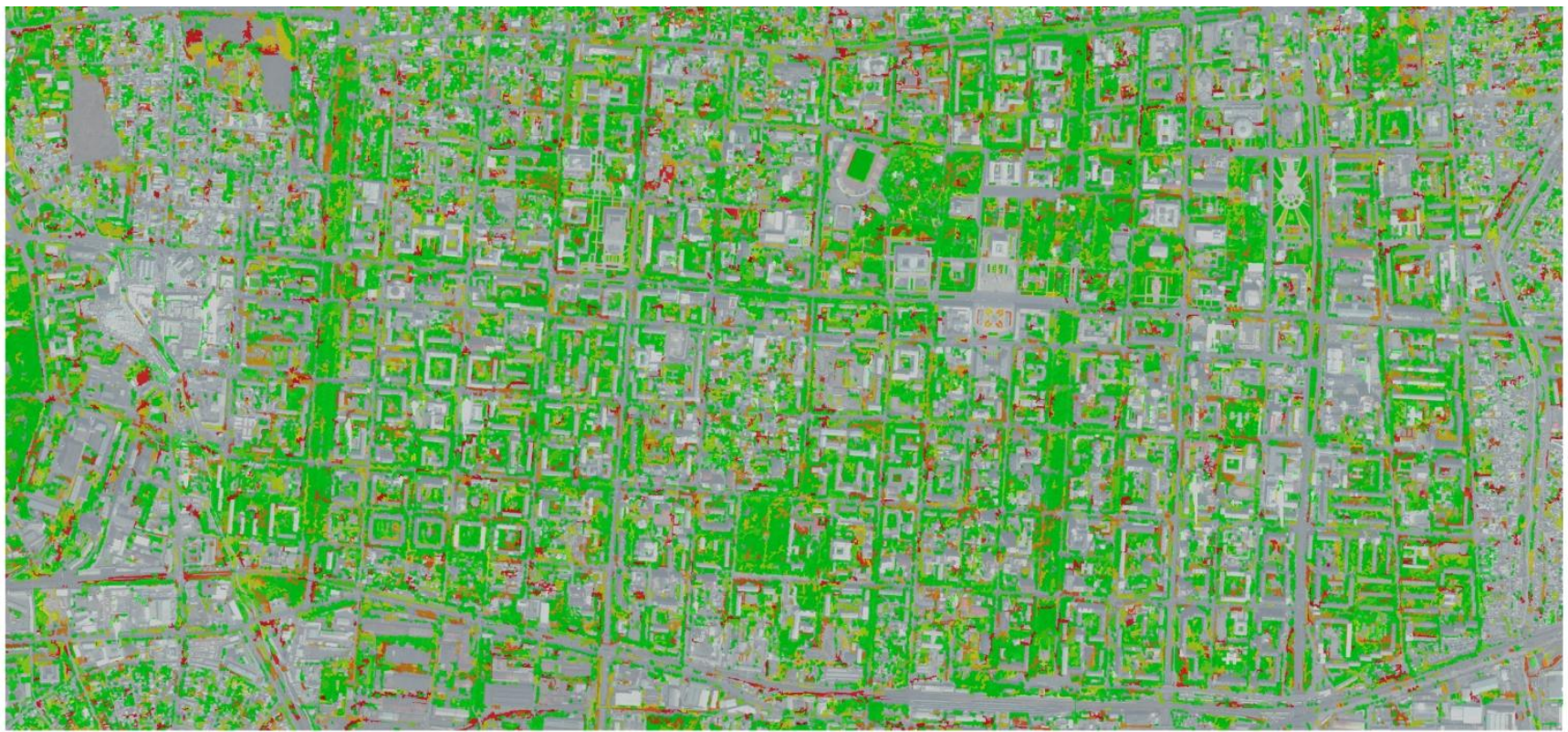

\begin{tabular}{|c|c|c|c|c|c|c|}
\hline \multirow{2}{*}{$\begin{array}{l}\text { Best } \\
\text { classification result }\end{array}$} & $0,10-0,19$ & $0,29-0,37$ & $0,47-0,55$ & $0,65-0,73$ & $0,83-0,91$ & \multirow[t]{2}{*}{ no vegetation } \\
\hline & $0,20-0,28$ & $0,38-0,46$ & $0,56-0,64$ & $0,74-0,82$ & $0,92-1,00$ & \\
\hline
\end{tabular}

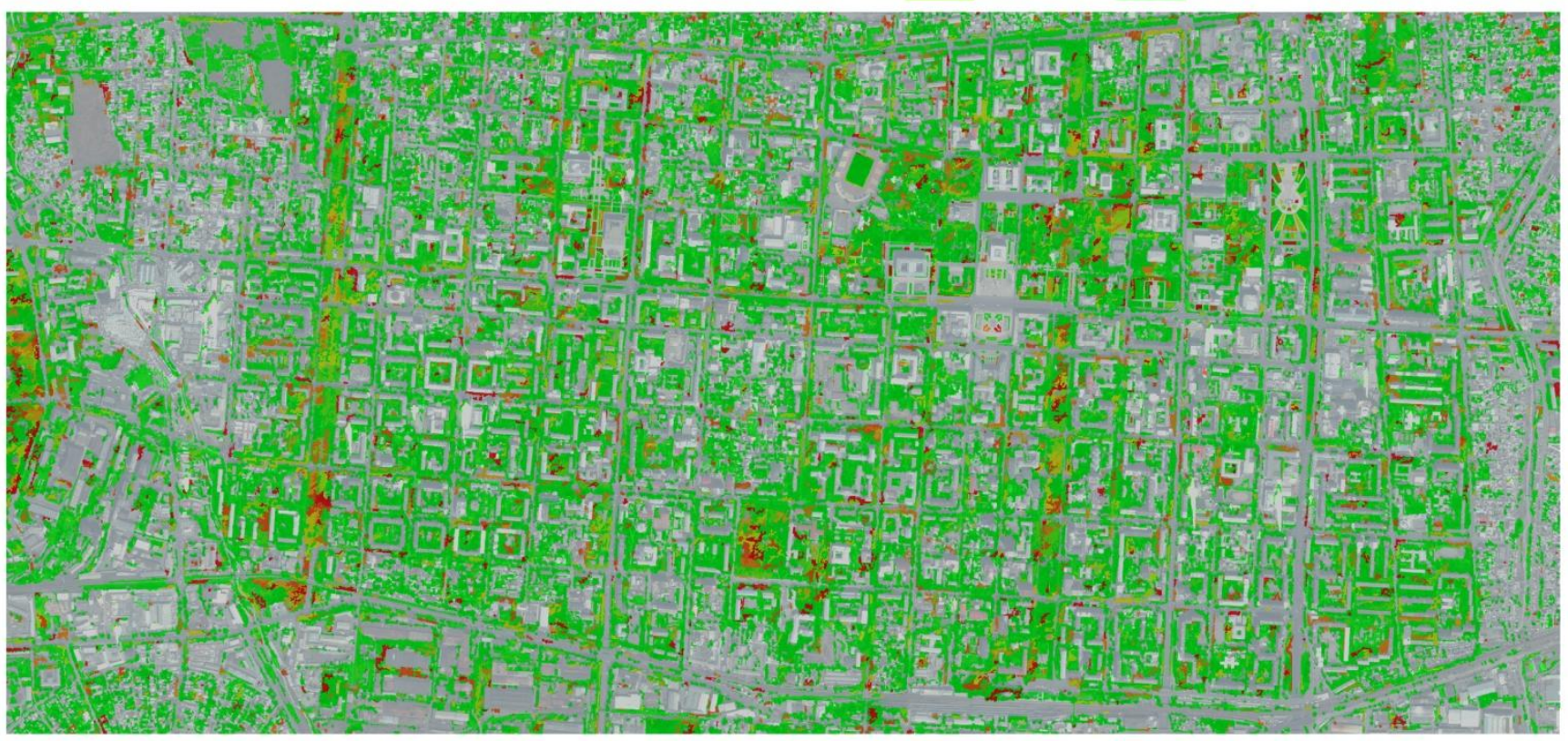

Classification Stability

$0,00-0,10$

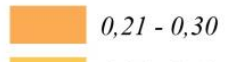

$0,11-0,20$

$0,31-0,40$

$0,41-0,50$

$0,51-0,60$

$0,61-0,70$

$0,71-0,80$

$0,81-0,90$

no vegetation

\section{Spatial Analysis and Mapping}

While image analysis produces a high resolution map of the land cover features of interest, to support longer-term monitoring as well as planning applications, a standardized geometry is desirable. Options are location-specific structures like micro districts or city blocks, or regular 'neutral' tilings like a regular grid. The latter is well suited as a common framework for integration of data sets from 
different sources and lends itself easily to a broad range of analysis techniques as well as visualization approaches. In the example present we have chosen a grid approach for further analysis of Bishkek's green spaces. Subsequent steps are based on a 100-m resolution (hectare) grid aligned with UTM.

\section{Developing an Urban Green Index}

In order to determine the 'Green Index' per cell following [4], first the vegetation polygons need to be intersected with grid cells. In contrast to [4] for the determination of the 'Green Index' per cell we have weighted the various types of vegetation differently. The 'Green Index' per cell $G I_{j}$ then is calculated by summarizing the weighted area $w_{c} A_{c}$ of the vegetation sub-classes $C$ within cell $j$ and dividing it by the area $A_{j}$ of the cell:

$$
G_{j}=\frac{1}{A_{j}} \sum\left(w_{C} A_{C}\right)_{j}
$$

With $0 \leq G I_{j} \leq 1$ and $0 \leq w_{c} \leq 1$. A 'Green Index' of $G I_{j}=0$ indicates no vegetation at all within cell $j$ and $G I_{j}=1$ indicates a complete coverage of the vegetation sub-class(es) weighted by 1 within cell $j$. In the example present we decided to weight the different sub-classes of vegetation as outlined in Table 4 .

Table 4. Class weights for the calculation of the Green Index.

\begin{tabular}{lc}
\hline Vegetation type & Weight \\
\hline meadow-like vegetation & 0.3 \\
mixed vegetation & 0.8 \\
wooded vegetation & 1.0 \\
\hline
\end{tabular}

Of course, these weights can be adjusted depending on the application framework. Results for the study area are presented in Figure 9.

\section{Impact of Classification Reliability on Analysis Results}

Having quantified information on the reliability of the input data, in principle allows assessing the reliability of subsequent spatial analysis. Spatial analysis results generated based on doubtful classification results can be highlighted or excluded from analysis. In order to evaluate the reliability of analysis results synoptically a cartographic presentation can be useful. Without going into detail about the visualization of uncertainty in maps [28] we decided to visualize the mean $\mu_{i}^{b}$ per cell as displayed in Figure 10. Of course $C S_{i}$ can be visualized accordingly. Alternatively, in order to avoid doubtful analysis results, unreliable or unstable objects can be excluded in advance from calculation of the 'Green Index'. For this purpose we decided to exclude objects (before intersecting with the grid cells) with a Classification Stability of $C S_{i} \leq 0.90$ and a Best Classification Result of $\mu_{i}^{b} \leq 0.75$ for the calculation of $G I_{j}$. Only vegetation objects fulfilling these criteria (Figure 10) are considered for calculating the weighted 'Green Index'. The difference between the $G I_{j}$ with and without reliable vegetation objects is relatively low - in the present subset we have observed a mean difference of 0.026 for the overall 'Green Index'. However, when excluding doubtful objects in advance, the reliability of the calculated $G I_{j}$ rises in many instances. 
Figure 9. Weighted Green Index superimposed to pan-sharpened GeoEye-1 image.

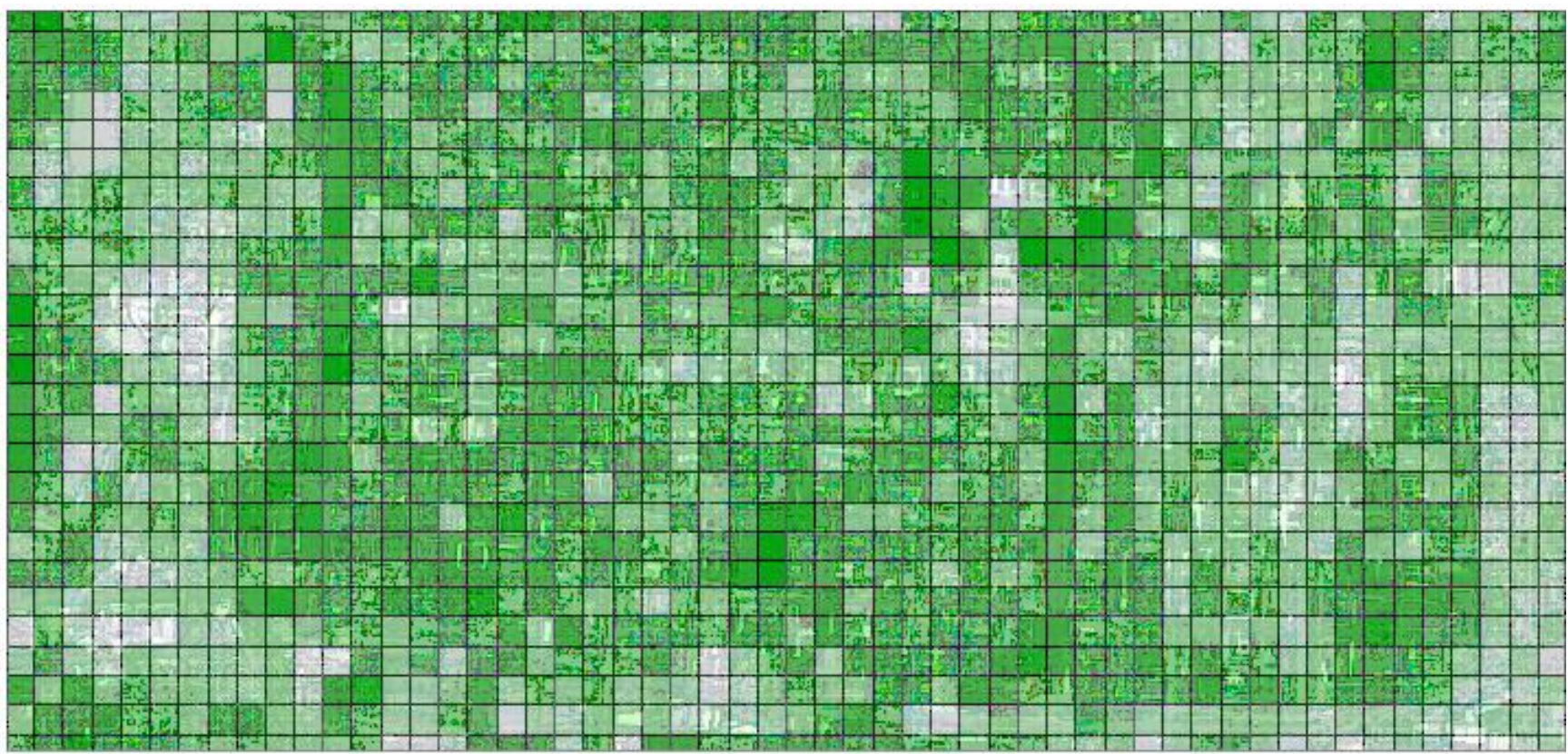

Weighted Green Index

\begin{tabular}{|l}
$\square$ \\
$0,16-0,24 \square$ \\
$\square, 24-0,32 \square$ \\
$0,40-0,47 \square$ \\
$0,47-0,55 \square$ \\
$0,32-0,40 \square$ \\
$0,0,71-0,79$
\end{tabular}

$0,00-0,08$

$0,32-0,40 \square 0,55-0,63$

Figure 10. Weighted Green Index superimposed on pan-sharpened GeoEye-1 image, plus mean Best Classification Result per cell as crosshairs. Size of crosshairs indicates the mean value of Best Classification Result per cell. Weighted Green Index and Best Classification Result are calculated based on vegetation objects with $C S_{i}>0.90$ and $\mu_{i}^{b}>0.75$. No crosshair indicates a Best Classification Result of $\mu_{i}^{b}>0.9$.

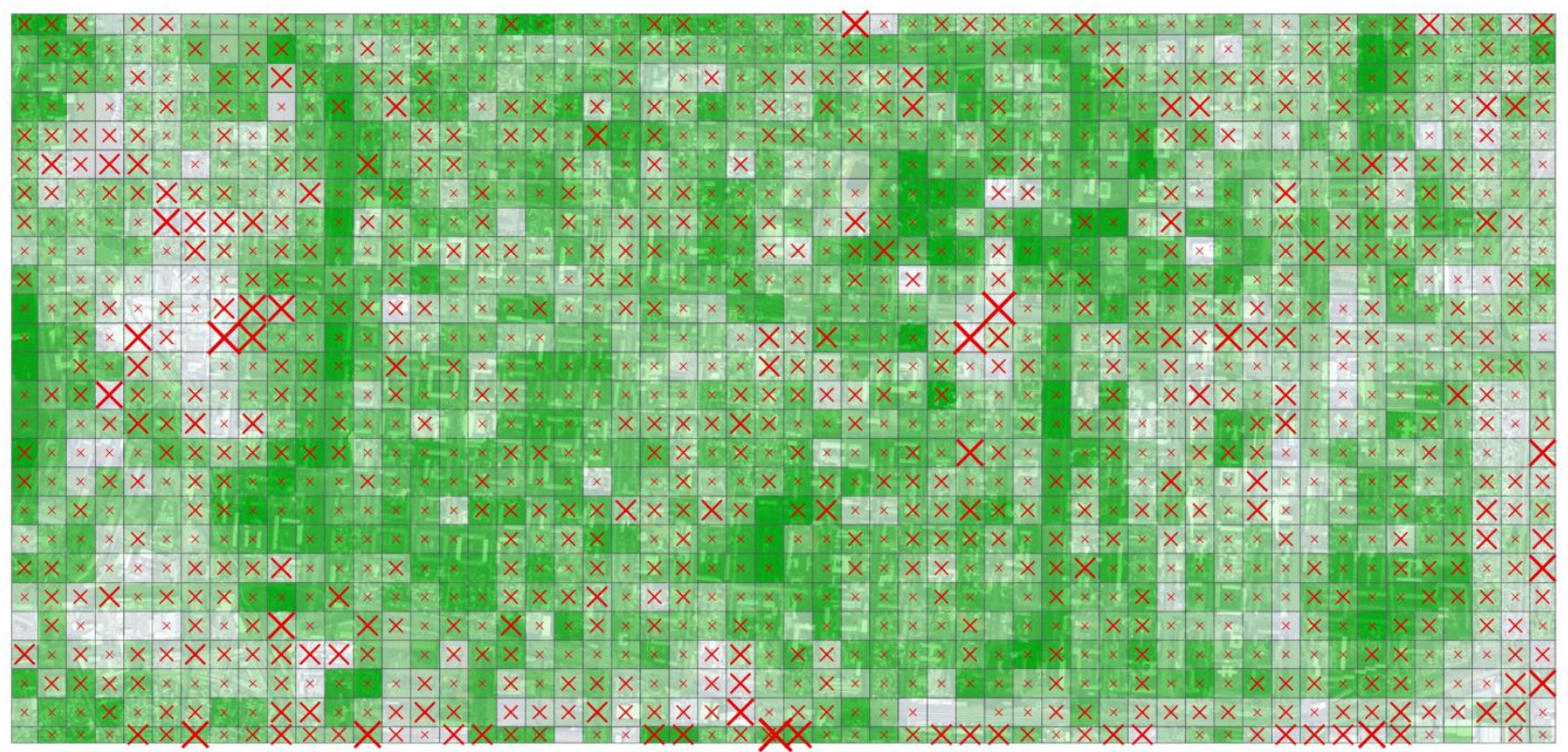

\section{Weighted Green Index}

$0,01-0,10$

$0,11-0,18$
$0,19-0,25$

$0,26-0,31$

$0,32-0,36$
$0,37-0,42$

$0,43-0,48$

$0,49-0,56$

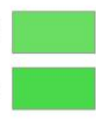

0,57 - 0,65 Mean Best Classification Result

$0,66-0,79$

$x$

$0,41-0,50$

$0,51-0,60$ $\times \quad 0,61-0,70$

$\times \quad 0,71-0,80$

$0,81-0,90$ 


\section{Results and Discussion}

This paper introduces a workflow for mapping a modified 'Green Index' as introduced by [4]. The modification is based on different weightings for vegetation classes determining the 'Green Index'. The weights presented here were chosen arbitrarily. Methodologically the paper focuses on estimating the reliability of classification results derived from object based image analysis and fuzzy classification. We demonstrate how primary classification reliability can be determined by the parameters Best Classification Result $\left(\mu_{i}^{b}\right)$ and Classification Stability $\left(C S_{i}\right)$ as introduced by [20], and implemented in the software package eCognition (see [26,27]). Both parameters are derived directly from fuzzy classification results. We further demonstrate how this information can be passed to the evaluation of reliability of subsequent spatial analysis (here: the calculation of a modified 'Green Index'). As outlined in Section $6, \mu_{i}^{b}$ and $C S_{i}$ can even be used to exclude obviously unreliably classified objects from further spatial analysis processes.

Nevertheless, we are aware that the parameters Best Classification Result $\left(\mu_{i}^{b}\right)$ and Classification Stability $\left(C S_{i}\right)$ are just comparing the classification results with their underlying class models. While $\mu_{i}^{b}$ shows how well a classified object fits a model, $C S_{i}$ expresses the ambiguity of the class assignment. However, none of the parameters expresses the consistency with reality, which still needs to be assessed by comparing classification results with on-site samples.

\section{Acknowledgments}

We gratefully acknowledge support by the GeoEye Foundation providing GeoEye-1 imagery for the city of Bishkek, a research fellowship awarded to Nazarkulova by the Eurasia-Pacific Uninet (http://www.eurasiapacific.net) and input from our fellow researchers at the Center for Geoinformatics, University of Salzburg.

\section{References}

1. Zoulia, D.; Santamouris, M.; Dimoudi, A.; Monitoring the effect of urban green areas on the heat island in Athens. Environ. Monit. Assess. 2009, 156, 275-292.

2. Alexandri, E.; Jones, P. Temperature decreases in an urban canyon due to green walls and green roofs in diverse climates. Build. Environ. 2008, 42, 480-493.

3. Robitu, M.; Musy, M.; Inard, C.; Groleau, D.; Modeling the influence of vegetation and water pond on urban microclimate. Solar Energy 2006, 80, 435-447.

4. Schöpfer, E.; Lang, S.; Blaschke, T. A "Green Index" Incorporating Remote Sensing and Citizen's Perception of Green Space. In Proceedings of the ISPRS Joint Conference on 3rd International Symposium Remote Sensing and Data Fusion Over Urban Areas (URBAN 2005) and 5th International Symposium Remote Sensing of Urban Areas (URS 2005), Tempe, AZ, USA, 14-16 March 2005; Volume 37, Part 5/W1, pp. 1-6.

5. Welch, R.; Ehlers, M.; Merging multiresolution SPOT HRV and Landsat TM Data. Photogramm. Eng. Remote Sensing 1987, 53, 301-303.

6. Lillesand, T.M.; Kiefer, R.W.; Chipman, J.W. Remote Sensing and Image Interpretation, 6th ed.; John Wiley \& Sons: Hoboken, NJ, USA, 2008. 
7. Lang, S. Object-based image analysis for remote sensing applications: modelling reality—Dealing with complexity. In Object Based Image Analysis; Blaschke, T., Lang, S., Hay, G.J., Eds.; Springer: Heidelberg/Berlin, New York, 2008; pp. 1-25.

8. Haralick, R.M.; Shapiro, L. Survey: Image segmentation techniques. Comput. Vis. Graph. Image Process. 1985, 29, 100-132.

9. Pal, N.R.; Pal, S.K. A review on image segmentation techniques. Patt. Recog. 1993, 26, 1277-1294.

10. Baatz, M.; Schäpe, A.; Multiresolution segmentation: An optimization approach for high quality multi-scale image segmentation. In Angewandte Geographische Informations-Verarbeitung; Strobl, J., Blaschke, T., Griesebner, G., Eds.; Wichmann Verlag: Karlsruhe, Germany, 2000; Volume XII, pp. 12-23.

11. Neubert, M.; Meinel, G.; Evaluation of Segmentation Programs for High Resolution Remote Sensing Applications. In Proceedings of the Joint ISPRS/EARSeL Workshop "High Resolution Mapping from Space 2003”, Hannover, Germany, 6-8 October 2003.

12. Hay, G.J.; Blaschke, T.; Marceau, D.J.; Bouchard, A. A comparison of three image-object methods for the multiscale analysis of landscape structure. ISPRS J. Photogramm. Remote Sens. 2003, 57, 327-345.

13. Neubert, M.; Herold, H.; Meinel, G. Assessing image segmentation quality-Concepts, methods and application. In Object Based Image Analysis; Blaschke, T., Lang, S., Hay, G.J., Eds.; Springer: Heidelberg/Berlin, Germany, 2008; pp. 760-784

14. Thiel, C.; Thiel, C.; Riedel, T.; Schmullius, C. Object-based classification of SAR data for the delineation of forest cover maps and the detection of deforestation-A viable procedure and its application in GSE forest monitoring. In Object Based Image Analysis; Blaschke, T., Lang, S., Hay, G.J., Eds.; Springer: Heidelberg/Berlin, Germany, 2008; pp. 327-343.

15. Lang, S.; Blaschke, T. Hierarchical Object Representation-Comparative Multiscale Mapping of Anthropogenic and Natural Features. In Proceedings of ISPRS Workshop "Photogrammetric Image Analysis”, Munich, Germany, 17-19 September 2003; Volume 34, pp. 181-186.

16. Hay, G.J.; Castilla, G.; Wulder, M.A.; Ruiz, J.R. An automated object-based approach for the multiscale image segmentation of forest scenes. Int. J. Appl. Earth Obs. Geoinf. 2005, 7, 339-359.

17. Koestler, A. The Ghost in the Machine; Random House: New York, NY, USA, 1967.

18. Câmara, G.; Souza, R.C.M.; Freitas, U.M.; Garrido, J. SPRING: Integrating remote sensing and GIS by object-oriented data modelling. Comput. Graph. 1996, 20, 395-403.

19. Lang, S.; Blaschke, T. Bridging Remote Sensing and GIS-What are the Main Supporting Pillars? In Proceedings of 1st International Conference on Object-based Image Analysis (OBIA 2006), Salzburg, Austria, 4-5 July 2006.

20. Benz, U.; Hofmann, P.; Willhauck, G.; Lingenfelder I.; Heynen, M.; Multi-resolution object-oriented fuzzy analysis of remote sensing data for GIS-ready information. ISPRS J. Photogramm. Remote Sens. 2004, 58, 239-258.

21. Liedtke, C.-E.; Bückner J.; Grau, O.; Growe, S.; Tönjes, R. AIDA: A System for the Knowledge Based Interpretation of Remote Sensing Data. In Proceedings of the Third International Airborne Remote Sensing Conference and Exhibition, Copenhagen, Denmark, 7-10 July 1997. 
22. Siler, W.; Buckley, J.J. Fuzzy Expert Systems and Fuzzy Reasoning; John Wiley \& Sons: Hoboken, NJ, USA, 2005.

23. Richards, J.A.; Jia, X. Remote Sensing Digital Image Analysis, 4th ed.; Springer: Berlin/Heidelberg, Germany, 2006.

24. Cristianini, N.; Shawe-Taylor, J. An Introduction to Support Vector Machines; Cambridge University Press: Cambridge, UK, 2000.

25. Zell, A.; Mamier, G.; Vogt, M.; Mache, N.; Hübner, R.; Döring, S.; Herrmann, K.-U.; Soyez, T.; Schmalzl, M.; Sommer, T.; et al. SNNS Stuttgart Neural Network Simulator, User Manual, Version 4.2, Available online: http://www.ra.cs.uni-tuebingen.de/downloads/SNNS/ SNNSv4.2.Manual.pdf (accessed on 15 May 2011).

26. Trimble. eCognition Developer 8.64.0 User Guide; Trimble: Munich, Germany, 2010.

27. Trimble. eCognition Developer 8.64.0 Reference Book; Trimble: Munich, Germany, 2010.

28. MacEachren, A.M.; Robinson, A.; Hopper, S.; Gardner, S.; Murray, R.; Gahegan, M.; Hetzler, E. Visualizing geospatial information uncertainty: What we know and what we need to know. Cartogr. Geogr. Inform. Sci. 2005, 32, 139-160.

(C) 2011 by the authors; licensee MDPI, Basel, Switzerland. This article is an open access article distributed under the terms and conditions of the Creative Commons Attribution license (http://creativecommons.org/licenses/by/3.0/). 